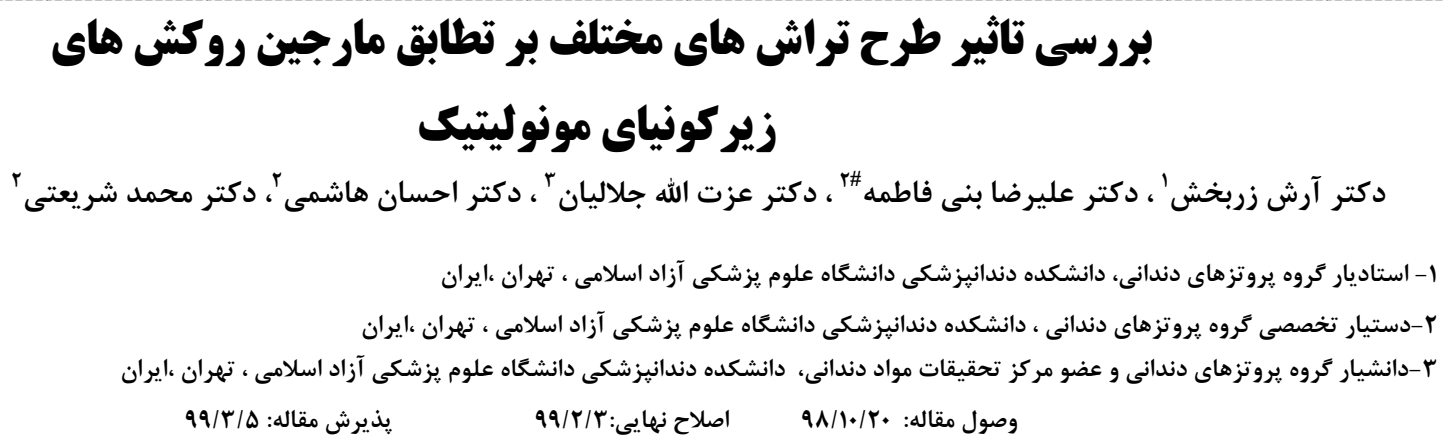

\title{
The Effect of Preparation Design on the Marginal Integrity of Monolithic Zirconia Crown
}

\author{
Arash Zarbakhsh ${ }^{1}$, Alireza Banifatemeh ${ }^{2}$, Ezzatollah Jalalian ${ }^{3}$, , Ehsan Hashemi ${ }^{2}$ \\ Mohammad Shariaty ${ }^{2}$
}

\footnotetext{
${ }^{1}$ Assisstant Prof, Department of Prosthodontics, Faculty of Dentistry, Tehran Medical Sciences, Islamic Azad University, Tehran, Iran

${ }^{2}$ Post Graduate Student, Department of Prosthodontics, Faculty of Dentistry, Tehran Medical Sciences, Islamic Azad University, Tehran, Iran

${ }^{3}$ Associate Prof, Department of Prosthodontics and Membership of dental material Faculty of Dentistry, Tehran Medical Sciences, Islamic Azad University, Tehran, Iran
}

Received: Dec 2020

; Accepted: May 2020

\section{Abstract}

Background \& Aim: Marginal adaptation of full ceramic zirconia restorations are one of the important factors in their long-term success and one of the factors affecting marginal adaptation and their strength is the finish line design. This study was conducted to compare the effect of finishline design on the adaptation of monolithic zirconia crowns.

Material and methods: In this in-vitro study, 3 standard PEEK dies with $7 \mathrm{~mm}$ height and $5 \mathrm{~mm}$ diameter (in cervical) with convergence angle of $10^{\circ}$ in the walls was prepared by Ceramill motion 2 (Amann Girrbach, Austria) in three groups with chamfer,sloped shoulder and shoulder design. After pouring the molds made from PEEK dies with epoxy resin,30 dies(10 samples per group) were made.The construction of zirconia crowns (zolid preshades,Amann Girrbach, Austria) was performed using the Ceramill map extraoral scanner (Amann Girrbach, Austria) and the CAD / CAM method Ceramill motion 2 (Amann Girrbach, Austria). After cementing the crowns with Panavia F2, they were cut in the Buccolingual direction and the gap was measured at 7 points by the SEM (TESCAN S8000, China) at 500x magnification. In this study, one-way Anova statistical methods were used to show the effect of preparation design on marginal matching and Tukey HSD test to compare groups with each other.

Results: Marginal gap values of the samples evaluated by the mean of AMO and MO,for Chamfer, Sloped shoulder and shoulder groups were $(60.37 \pm 11.78 / 57.29 \pm 12.63),(73.37 \pm 14.79 / 68.08 \pm 14.21)$ and (95.42 $\pm 9.60 / 92.59 \pm 10.83)$ microns and the internal gap values measured by Axial and Occlussal averages were $(49.60 \pm 10.37 / 120.37 \pm 33.61),(46 \pm 9.37 / 124.41 \pm 32.10)$ and $(69.43 \pm 9.73 / 163.49 \pm 27.79)$ microns respectively. There was a significant difference between Chamfer and sloped shoulder groups with shoulder group $(\mathrm{P}<0.05)$ but there was no significant difference between Chamfer and sloped shoulder group $(\mathrm{P}>0.05)$.

Conclusions: From the results,it may be concluded that chamfer and sloped shoulder showed better marginal adaptation than that of shoulder,although the chamfer group had the better adaptation among all groups.

Key words: Dental Marginal adaptation, zirconia, Dental Crown

\# Corresponding Author: a.banyfatemeh@gmail.com

J Res Dent Sci. 2020; 17 (2): 107-115 
سابقه و هدف: تطابق لبه اى رستوريشنهاى تمام سراميك زيركونيايى يكى از عوامل مههم موفقيت طولانى مدت آنها محسوب ميشود

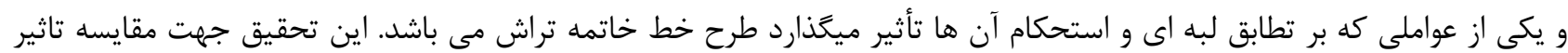

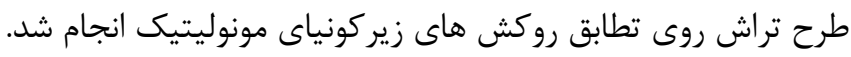

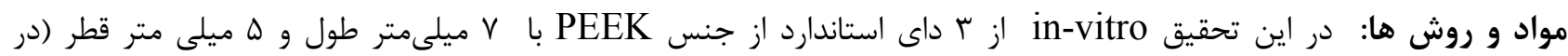

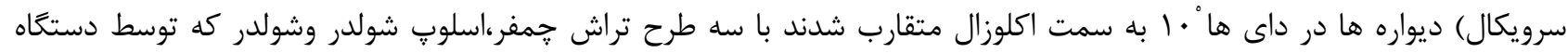

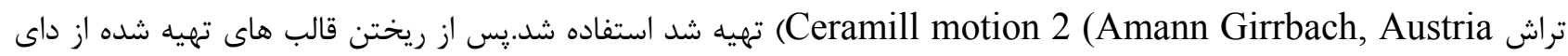

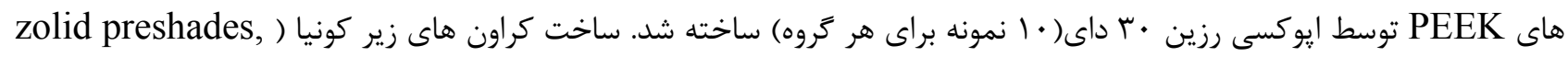
(Amann Girrbach, Austria CAD/CAM Ceramill motion 2 (Amann Girrbach, Austria

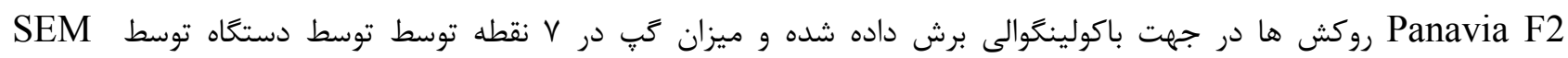

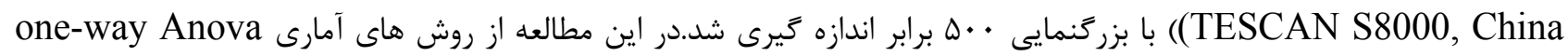

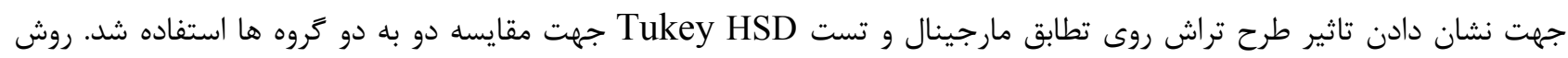
امارى ذكر شود

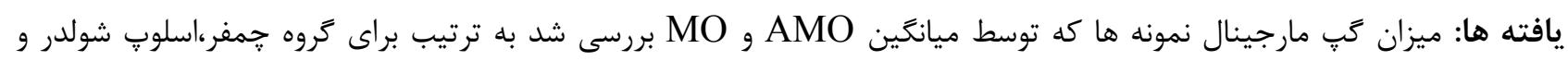

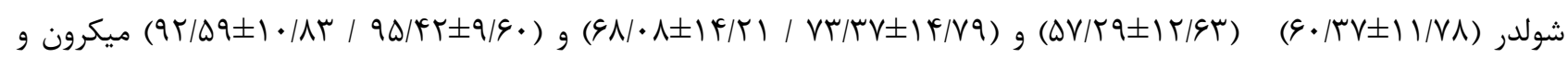

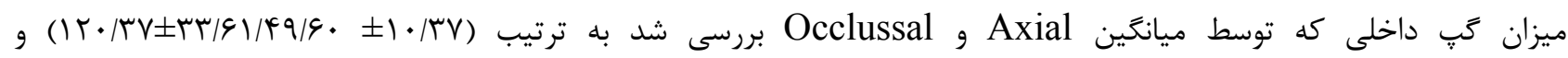
(

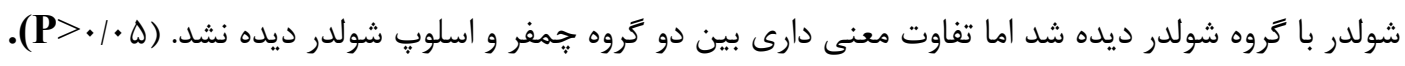

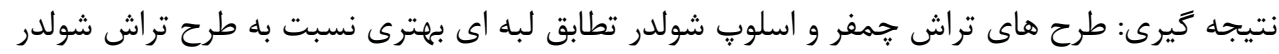

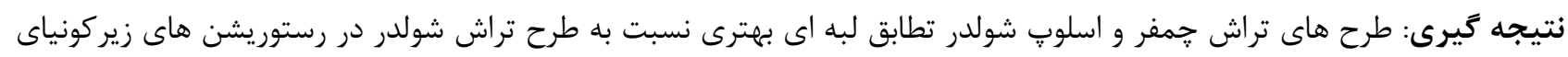

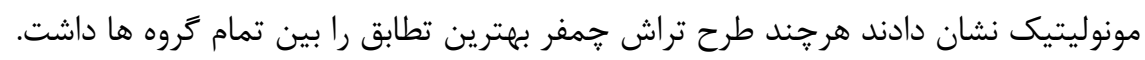

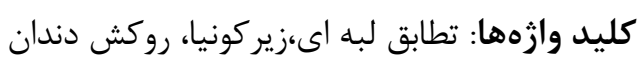

in-vivo التهاب در پالب وايتال فراهم مى گردد. (r) مطالعات

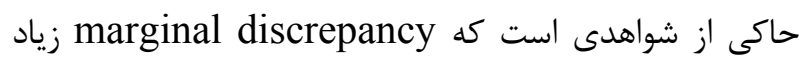
در يك رستوريشن ثابت با plaque index بالاتر و اثرات مخرب يربودنتال در ارتباط است (ه-") اخيرا تحقيقى نشان داد

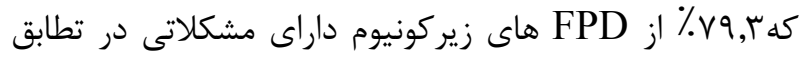

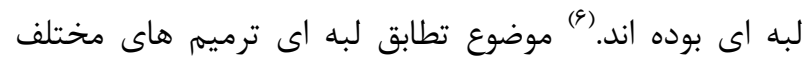

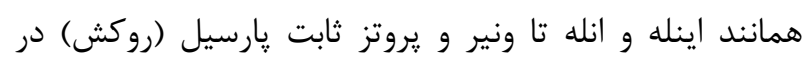

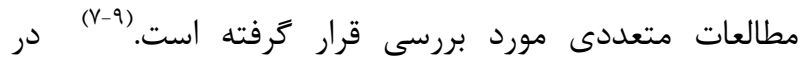

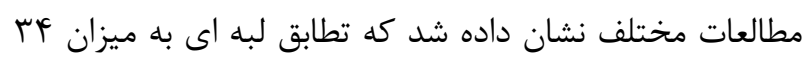

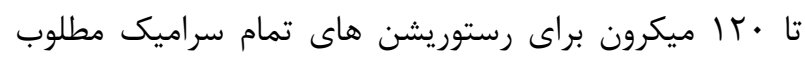

موفقيت يك رستوريشن دندانى، اساسا توسط سه عامل تعيين مى شود: زيبايى، مقاومت به شكست و تطابق لبه اى (marginal fit)

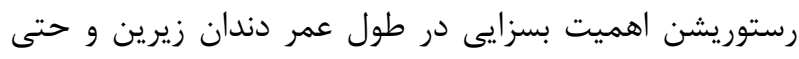

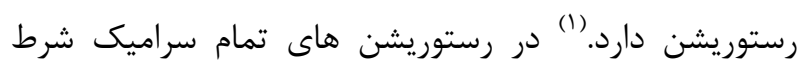

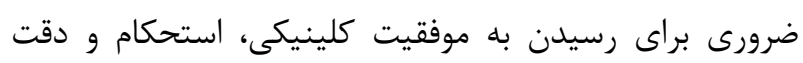
تطابق لبه اى است.افزايش marginal discrepancy منجر برون

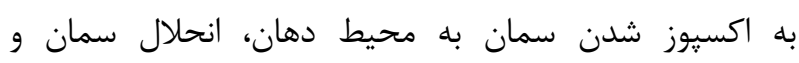
ميكروليكيج مى گردد. در نتيجه، سيل سمان تضعيف شده و وانه

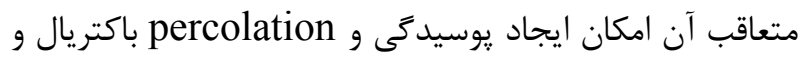


متقارب شدند(براى هر ديواره هُ) . خط انتهاى تراش در داى ها

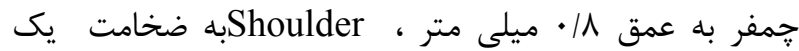

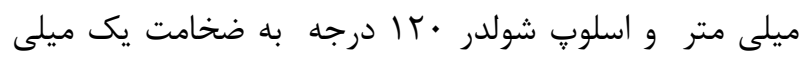
متر در نظر كرفته شد. (19) (شكل (1) متربل

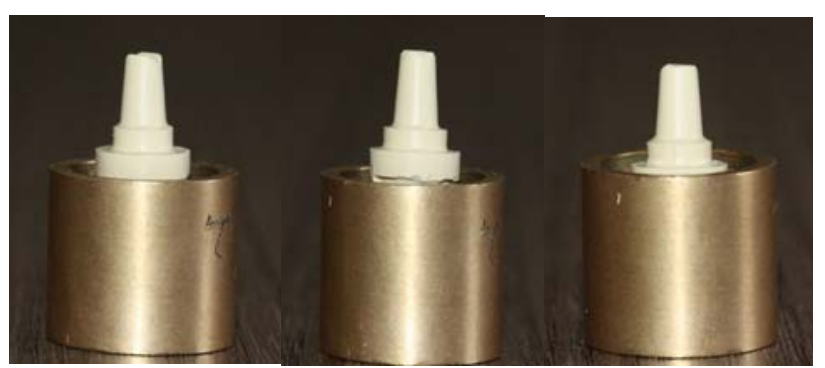

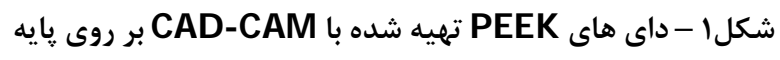

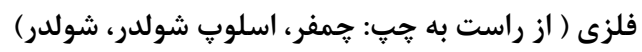

داى استاندارد سيس بر روى يك پايهى فلزى ثابت شد و قالب كيرى از اين داى توسط ترى اختصاصى استينلس استيل داراى يك عدد سوراخ در اكلوزال با استاٍ سرويكالى با داى كه فضاى لرى يكنواخت ه/ ميلى متر داخل آن ايجاد شده انجام كرفت.به منظور قالب بPanasil, kettenbach, Germany) مرحلهاى استفاده شد.قالبگيرى براى هر داى • ا بار تكرار

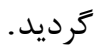

يس از Set شدن ماده، قالب را از روى داى برداشته و تمامى

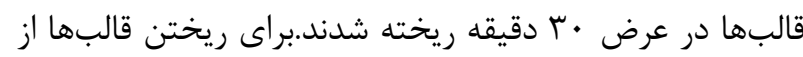
ايوكسى رزين (CW2215 Hunstman-Germany) استفاده

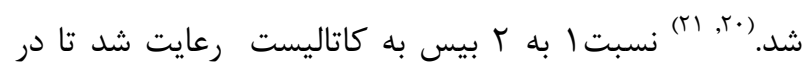
نهايت استحكام كافى و خصوصيات إيتيمم داىهاى نمونه حفظ شود. در نهايت · r عدد داى ايوكسى رزين ساخته شد.(تمامى اين مراحل در دماى معين اتاق انجام مى شود و به علت وضعيت يايه ى داى نيازى به dich نمونه ها نبود.
محسوب ميشود(|'·') مارجينال گֶٍ(عدم تطابق مارجين) در روكش هاى تمام سراميك علاوه بر فرآيند ساخت و طرح

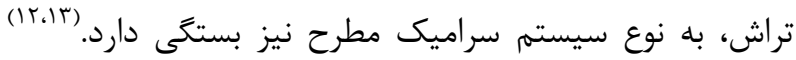

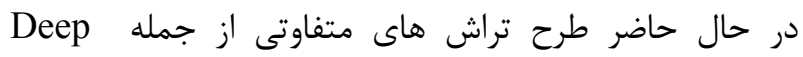
براى آماده سازى دندان ها Shed Shoulder Chamfer و ساخت رستوريشن هاى زيركونيايى ونير شونده مورد استفاده قرار مى گيرند ،هرجند مشكلاتى در مورد روش ساخت روكش هاى زيركونيايى ونير شونده وجود دارد، براى مثال گزارشات متعددى از يريدگى ونير يرسلن فلدسياتيك،باعث ايجاد توجه

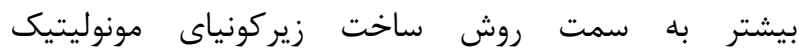
شدهمجنين زير كونياى مونوليتيك با توجه به عدم وجود لايه ى ونير و همجنين استحكام شكست كافى مى توانند به راحتى رئى در نواحى دندان هاى خلفى كه تحت نيروى اكلوزال بيشترى

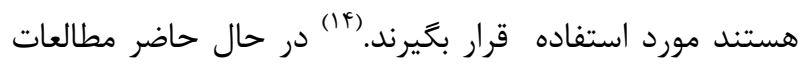
متعددى در مورد تاثير تراش هاى مختلف بر روى تطابق رستوريشن هاى زيركونيايى ونير شونده انجام گرفته كه نتايج

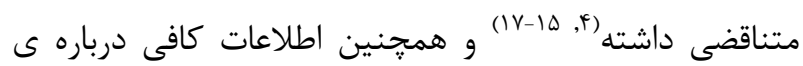
جَكَنَّى تاثير اين طرح تراش ها بر تطابق لبه اى اين رستوريشن هاى زيركونياى مونوليتيك در دست نيست.با توجه به تناقضات مطرح شده و نيز عدم وجود مطالعات قبلى در مورد تطابق لبه اى زيركونياى مونوليتيك و محدوديت هاى تحقيقات قبلى و نيز با توجه به اهميت موضوع و خلا اطلاعاتى، در اين مونى تحقيق تطابق لبه اي در رستوريشن هاى زيركونياى مونوليتيك با طرح تراش اسلوب شولدر،جمفر كلاسيك و راديال شولدر مورد بررسى قرار كرفت. مواد و روش ها: در اين تحقيق كه به صورت in-vitro انجام

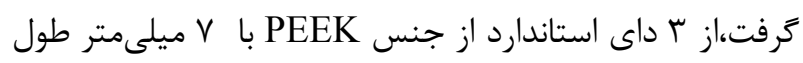

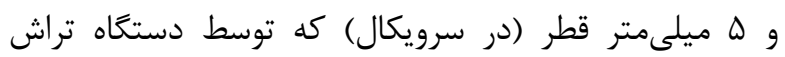
تمييه Ceramill motion 2 (Amann Girrbach, Austria) شد استفاده شد.(1) ديواره ها در داى هاء · ل به سمت اكلوزال

مجله تحقيق در علوم دندانيزشكى/دوره هفدهم /شماره دوم /تابستان 99 إيبايى 
سيس كليه نمونه ها درون آكريل مانت شده و عمل برش توسط ديسك هاى مخصوص لابراتوارى (speedy,prodont (Hollinger,france absolute marginal عمودى انجام كرفت.سيس ميز marginal opening ور دو نقطه از لبه هاى opening تراش(point 1,2,6,7) و ميزان internal fit در سه ناحيه باكال،لينغوال و اكلوزال(point 3,4,5) توسط دستخاه توسط ه. (SEM (TESCAN S8000, China

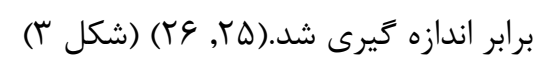

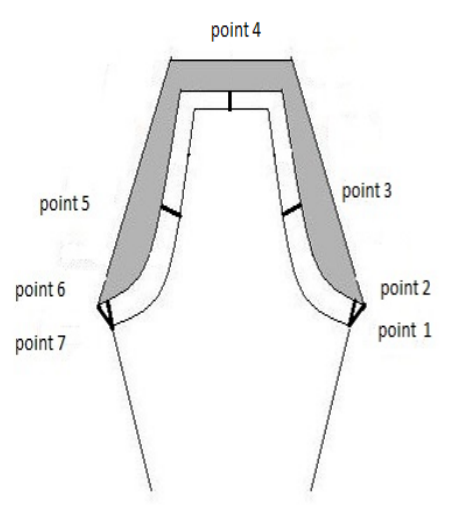

شكل r- نماى شماتيك انتخاب نقاط اندازه كَيرى براى ميزان point ) marginal opening و absolut marginal opening (point 3,4,5) internal fit (1,2,6,7 و ميزان

AMO(absolute عيس ميانگين نقاط اوV براى marginal opening MO(marginal opening Occlussal Axial Internal fit Internal fit

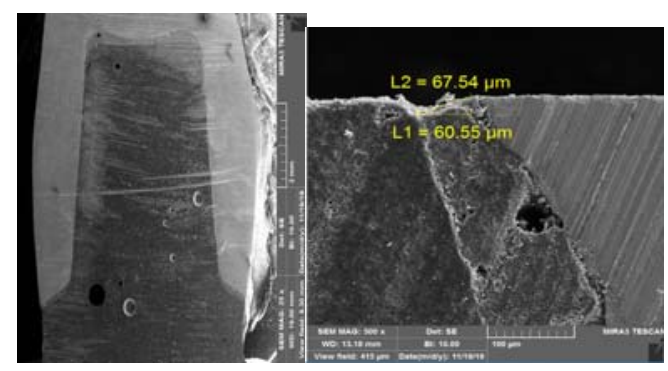

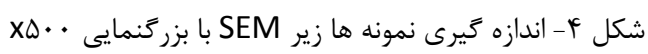

سِّ مراحل ساخت كراون ها شروع شد.ساخت كراون هاى

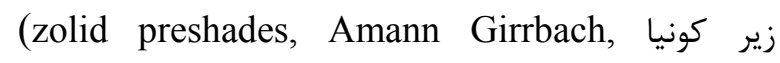
Ceramill map بustria) (Amann Girrbach, Austria) ريليف به ميزان هـ ميكرون با فاصله يك ميليمترى از لبه
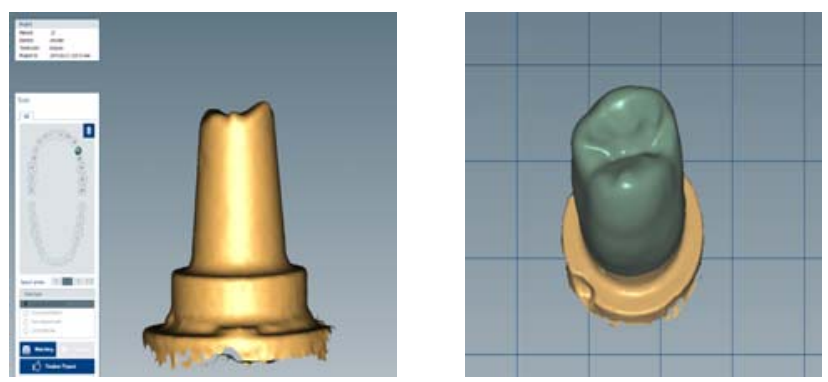

تراش كه با استفاده از نرم افزار اين مقدار اعمال مى شود و

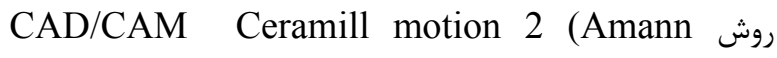
Girrbach, Austria)

شكلץ - اسكن و طراحى كراون ها با نرم افزار CAD-CAM

اين ساختارهاى mill شده را از دستخاه خارج كرده و در قرار

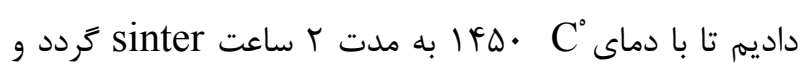

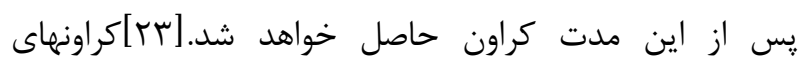
حاصله به منظور افزايش قدرت سمان توسط ذرات آلومينيوم

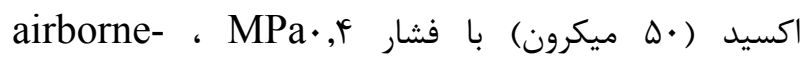
شدند سيس كراونها روى داى خود قرار كرفته و شمارهگذارى شدند.

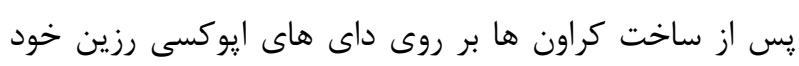

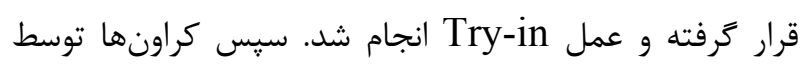
سمان دوال كيور (Panavia F2, Kuraray, Japan) توسط دستگاه يرس به مدت • ا دقيقه تحت نيروى عمودى ه ه

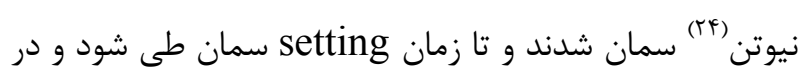

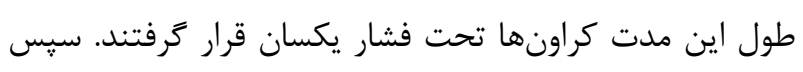

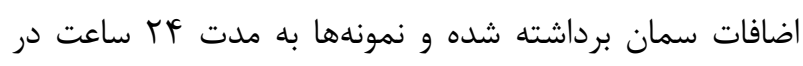
دماى اتاق در داخل نرمال سالين نكَهدارى شدند. 


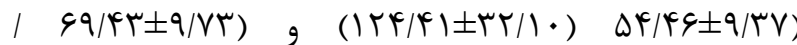

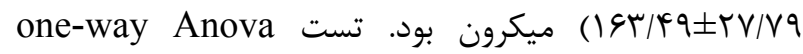
نشان داد نوع طرح تراش تاثير معنى دارى بر روى گٍ $\operatorname{Axial}(\mathrm{p}<0.001)$ ، $\mathrm{MO}(\mathrm{P}<0.001)$ ، $\operatorname{AMO}(\mathrm{P}<0.001)$

و Occlusal $(p=0.008)$ در r گروه داشت.(جدول l)

تست Tukey HSD بين گروه ها دو به دو انجام شد و اختلاف معنى دارى در گٍ

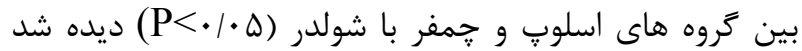
ولى اختلاف معنى دارى بين گروه هاى اسلوٍ شولدر و جمفر مشاهده نخرديد.(جدول r)
در اين مطالعه از روش هاى آمارى one-way Anova جهت نشان دادن تاثير طرح تراش روى تطابق مارجينال و تست جukey HSD

$$
\text { شد.روش امارى ذكر شود }
$$

\section{يافته ها:}

يس از بررسى توزيع نرمال داده هاى گروه ها توسط تست One-Sample Kolmogorov-Smirnov داده ها انجام گرفت.ميزان گٍَ مارجينال نمونه ها كه توسط ميانگين AMO و MO بررسى شد به ترتيب براى گروه

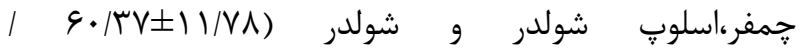

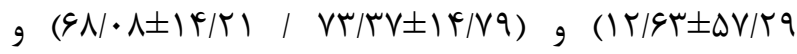

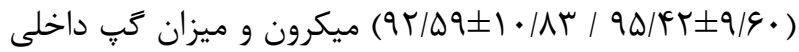
كه توسط ميانگين Axial و Occlussal بررسى شد به ترتيب (I ) • (

one-way anova جدول ا- نتيجه آناليز

\begin{tabular}{|c|c|c|c|c|c|c|c|c|c|}
\hline \multirow{2}{*}{ حداكثر } & \multirow{2}{*}{ حداقل } & \multicolumn{2}{|c|}{$\begin{array}{c}\mathrm{q}^{0} \% \text { Confidence Interval } \\
\text { for Mean }\end{array}$} & \multirow{2}{*}{$\begin{array}{l}\text { Std. } \\
\text { Error }\end{array}$} & \multirow{2}{*}{$\begin{array}{c}\text { Std. } \\
\text { Deviation }\end{array}$} & \multirow{2}{*}{ Mean } & \multirow{2}{*}{ تعداد } & \multirow{2}{*}{ تراش } & \\
\hline & & $\begin{array}{l}\text { Upper } \\
\text { Bound }\end{array}$ & $\begin{array}{l}\text { Lower } \\
\text { Bound }\end{array}$ & & & & & & \\
\hline qז.V^ & 19.11 & $\Delta r . q \Delta \Lambda V$ & gr.varr & f.gVAlf & If.VATهA & $V r / T r$ & 1. & اسلوب & \multirow{4}{*}{$\mathrm{AMO}$} \\
\hline 111.11 & V9.५ร & 1.т.ravr & $M . \Delta F \cdot V$ & Vופץ.r. & $9.9 \cdot|r|$ & $90 / 4 T$ & 1. & شولدر & \\
\hline W. $.9 \Lambda$ & rו. & 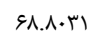 & $01.94 \wedge 9$ & T.VTATr & $11 . V \Lambda \cdot r r$ & $s \cdot / r V$ & 1. & جمفر & \\
\hline 111.11 & r r & $\Lambda r . \mu F \cdot s$ & $99 . r \varphi g V$ & T.FFADF & M.AVT & $v \varepsilon / v^{\prime} q$ & r. & كل & \\
\hline M.FV & rQ.r & VA.TQ.r & dV.qIVr & r.pqr=D & IE.rIIFA & 91.11 & 1. & اسلوب & \multirow{4}{*}{ MO } \\
\hline 11. & $V \cdot .9 F$ & I... & $\Lambda F . \Delta F \& \Lambda$ & r.FYs८q & 1..人ץqvq & Qr.बq & 1. & شولدر & \\
\hline V1. $.9 q$ & r. & 99.rтqV & FA.TAFT & r.१9019 & 1r.grrq & $\Delta V . r q$ & 1. & קمفر & \\
\hline 11. & r..1 & va.мus & שTאF & T.DTTVE & 19.requr & VT. . D & $r$. & كل & \\
\hline VI.ve & $r 0.91$ & $91.19 N 8$ & FV.VAYF & r. 99010 & १.TYGGF & $\Delta F . E S$ & 1. & اسلوٍ & \multirow{4}{*}{ Axial } \\
\hline$\wedge r$ & $\Delta T_{.} \cdot{ }^{r}$ & $v \xi . r \cdot \Delta \Delta$ & GT.FVTD & $r . \cdot \vee Q \Delta q$ & ৭.VTNAT & 99.4 & 1. & شولدر & \\
\hline 99.49 & re.g & $\Delta V \cdot \cdot r \cdot r$ & RT.UAT & $r . r \wedge|\Delta|$ & $1 . . r V V \cdot 9$ & pq.8. & 1. & جمفر & \\
\hline$\Lambda r$ & $\mu r .5$ & ST.SIQT & $\Delta r . \Delta S \mid$ & r.Trs9ם & Ir.A & $\Delta V . \wedge r$ & $\mu$. & كل & \\
\hline$r \cdot 0.91$ & $94 . T \Delta$ & IfY.rYAq & 1.1 .4911 & $1 . . \Delta Q T \Delta S$ & $r r . I \cdot \lambda r r$ & $|r F . F|$ & 1. & اسلوب & \multirow{4}{*}{ Occlusal } \\
\hline MIQ.AV & ITH.Ar & IAT.rVID & 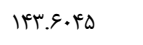 & A.VAG\&Y & TV.VQDTr & 195.199 & 1. & شولدر & \\
\hline $11 \cdot . \Delta 9$ & $11 . \cdot \Delta$ & IFF.FT.V & Q૬.rTVץ & $1 . .9 r$ & rr.s1Q & $M \cdot . r V$ & 1. & جمفر & \\
\hline$r \mid Q . A V$ & $11 . \cdot \Delta$ & $1199 . \Delta 510$ & $1 \pi r .9191$ & 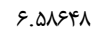 & TE.•VAGT & 145.99 & r. & كل & \\
\hline
\end{tabular}

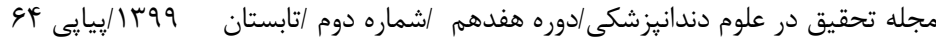


جدول r- نتيجه آناليز تست Tukey HSD

\begin{tabular}{|c|c|c|c|c|c|c|}
\hline \multirow{2}{*}{ 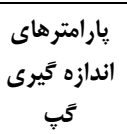 } & \multirow{2}{*}{\multicolumn{2}{|c|}{ مقايسه كروه هاى تراش }} & \multirow[b]{2}{*}{ Std. Error } & \multirow[b]{2}{*}{ Sig. } & \multicolumn{2}{|c|}{$৭ ৯ \%$ Confidence Interval } \\
\hline & & & & & $\begin{array}{l}\text { Lower } \\
\text { Bound }\end{array}$ & $\begin{array}{l}\text { Upper } \\
\text { Bound }\end{array}$ \\
\hline \multirow{6}{*}{ AMO } & \multirow{2}{*}{ اسلوب } & شولدر & $\Delta . F V G \cdot \Delta$ & $\ldots 1$ & $-r \Delta . q \mu \cdot r$ & $-\wedge . F \vee \Delta S$ \\
\hline & & جمفر & $\Delta . F V \varepsilon \cdot \Delta$ & سو.. &.$- \Delta V V F^{e}$ & TE. DVYF \\
\hline & \multirow{2}{*}{ 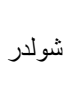 } & اسلوبٍ & $\Delta . F V G \cdot \Delta$ & $\ldots 1$ & N.FVAS & 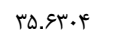 \\
\hline & & جمفر & $\Delta . F V G \cdot \Delta$ & $\ldots$ & TI. FVDS & FA.gr.t \\
\hline & \multirow{2}{*}{ جمفر } & اسلوب & $\Delta . \mp V \varepsilon \cdot \Delta$ & 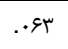 & $-r \varepsilon . \Delta V V^{e}$ &.$\Delta V V^{e}$ \\
\hline & & 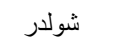 & $\Delta . F V G \cdot \Delta$ & $\cdots$ & 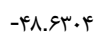 & - TI.FVDS \\
\hline \multirow{6}{*}{ MO } & \multirow{2}{*}{ اسلوب } & 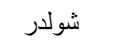 & 0.901 .9 & $\cdots 1$ & 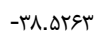 & $-1 \cdot .0 \cdot T V$ \\
\hline & & جمفر & 0.901 .9 & $.1 \Delta \Delta$ & מצוזי & M.人.M M \\
\hline & \multirow{2}{*}{ 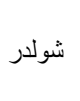 } & اسلوب & 0.901 .9 & $\cdots 1$ & $1 \cdot .0 \cdot r V$ & 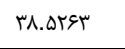 \\
\hline & & جمفر & 0.901 .9 & $\ldots$ & rI.rqQV & rq.rikr \\
\hline & \multirow{2}{*}{ جمفر } & اسلوب & 0.901 .9 & $.1 \Delta Q$ & שr.N. & r.Tाq \\
\hline & & شولدر & 0.901 .9 & $\ldots$ & سגוس. & -rI.raDV \\
\hline \multirow{6}{*}{ Axial } & \multirow{2}{*}{ 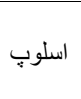 } & 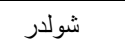 & 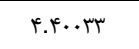 & $\cdots 9$ & -rD.MMN & $-4.9 \mathrm{VV}$ \\
\hline & & جمفر & 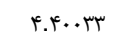 &.$\Delta T$. & $-9 . \cdot \Delta \& \mu$ & 10.VAfer \\
\hline & \multirow{2}{*}{ 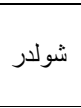 } & اسلوب & F.t...r & $\ldots 9$ & $4 . .9 \mathrm{VV}$ & rD.MMN \\
\hline & & جمفر & f.f..rm & $\ldots$ & 1.9TIV & אזאV.•" \\
\hline & \multirow{2}{*}{ جمفر } & اسلوب & f.f...r & . Dr. & 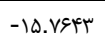 & $9 . \Delta Q Y$ \\
\hline & & شولدر & r.t. & $\ldots$ & מזאט. ש- & $-\Lambda .9 \mathrm{TIV}$ \\
\hline \multirow{6}{*}{ Occlusal } & \multirow{2}{*}{ اسلوب } & شولدر & 1r.qufeq &.$r \Delta$ & $-V \Psi \cdot V \Delta / f$ & $-f . t \cdot t g$ \\
\hline & & جمفر & 1 Ir.qufeq & $.9 \Delta \Delta$ & -r.grve & rN.V.qץ \\
\hline & \multirow{2}{*}{ شولدر } & اسلوبٍ & $11.91 F f q$ &.$r \Delta$ & $f . f+18$ & Vr.valf \\
\hline & & جمفر & $1 \% . q 1 \times 4 q$ & r & $1.4 F \cdot \varepsilon$ & VV.VAVE \\
\hline & \multirow{2}{*}{ جمفر } & اسلوبٍ & $1 \% . q \wedge<4 q$ & $.9 \Delta \Delta$ & $-r \Lambda . v \cdot q r$ & $r \cdot . s r v e$ \\
\hline & & شولدر & $1 \% . q 14 \mathrm{feq}$ & r & -VV.VAVF & $-1.44 \cdot 9$ \\
\hline
\end{tabular}

ميكروسكوף SEMاندازه گيرى شد.ميزان مارجينال گٍ براى كروه HTZ حدود(H/DV

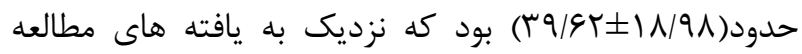
فعلى مى باشد. (ז^) در مطالعه in-vitro انجام شده،تطابق لبه اى توسط يارامترهاى AMO و MO وتطابق داخلى توسط اندازه گيرى تطابق axial و occlusal مشخص شد.AMO در اكثر مطالعات جهت بررسى تطابق لبه ای استفاده مى شود.با توجه به اين كه اندازه كيرى AMO با توجه به underextension وركش ها صورت مى overextention گيرد"(")، ممكن است بنا به انتخاب نقاط اندازه گيرى مختلف
تطابق لبهاى رستوريشنهاى تمام سراميك زيركونيايى يكى از عوامل مهرم موفقيت طولانى مدت آنها محسوب ميشود و يكى از عواملى كه بر تطابق لبه اى و استحكام آن ها تأثير ميخذارد

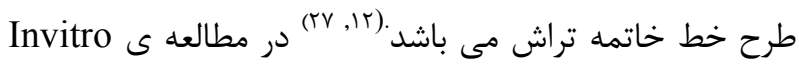
انجام شده توسط Rayyan در سال 19 • r كه مبنى بر مقايسه تطابق لبه اى دو گروه كويينگ هاى زيركونياى با ونيرينَ يرسلن و زيركونياى مونوليتيك با ترانسلوسنسى بالا(HTZ) بود,هر گروه توسط اسكن داى استاندارد با فينيش لاين جمفر كلاسيك با تعداد نمونه · ا عدد براى هر گروه در نظر گرفته شد.ميزان مارجينال گٍ عمودى در 1 نقطه توسط 
كه با يافته هاى اين مطالعه در مورد MO كروه شولدر با ميانخين 9 ه, ب9 همخوانى دارد. با اين حال تحقيقاتى نيز وجود دارند كه اعداد متفاوتى براى

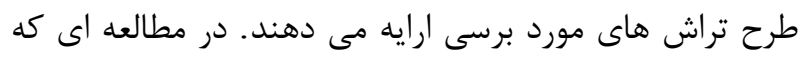
Jalalian

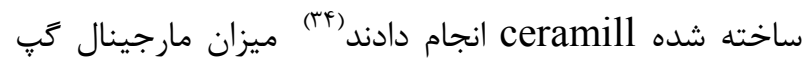

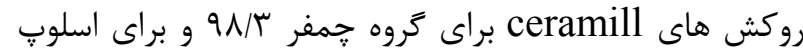

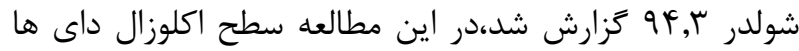
به صورت غير آناتوميك و مسطح در نظر كرفته شده بود كه مى تواند دليل اختلاف با مطالعه ى فعلى باشدهمينهنين

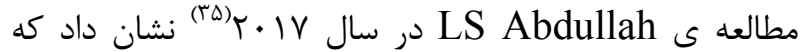
طرح تراش جمفر با سطح اكلوزال آناتوميك تطابق لبه اى لى بهترى(38.837) نسبت به سطح مسطح(63.199) داشته و در مورد طرح تراش شولدربرعكس مى باشد(66.63 براى بهرئ

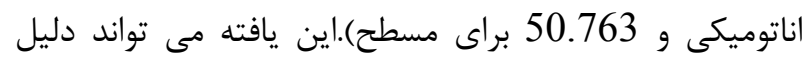
متفاوت بودن يافته هاى مطالعه Jalalian با مطاله ما را توجيه

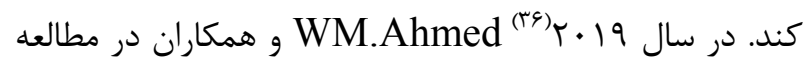
اى كه بر روى تاثير نحوه سينترينَ و طرح خاتمه تراش روى تطابق مارجينال روكش هاى مونوليتيك ساخته شده روى اباتمنت هاى تيتانيومى كه توسط سيستم Atlantis اسكن و ساخته شده بودند انجام گرفت نشان داد كه در بين گروه هايى

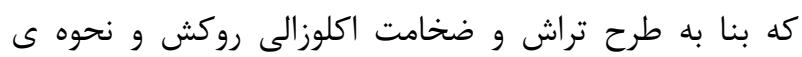
سينترينگ استاندارد و سريع در با كروه طبقه بندى شده بودند بهترين تطابق لبه ایى عمودى مربوط به كروه با گروه

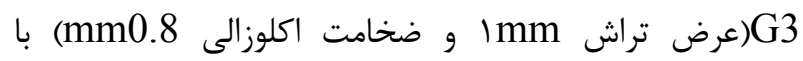
ميانگين سَF, IF ميكرون و كمترين تطابق لبه اى مربوط به كروه G8(عرض تراش هmm, · و ضخامت اكلوزالى هmm, () ) با ميانگين PV,qه بود.با توجه به نزديك بودن معيارهاى كروه

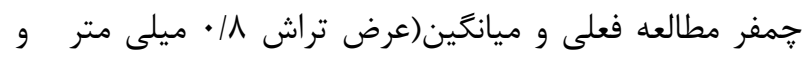

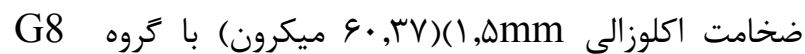

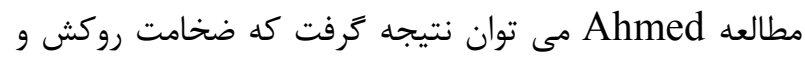
نحوه سينترينگ روى تطابق تاثير مى كذارد.
اعداد به دست آمده در مطالعات متفاوت باشدهمجنين به دليل لبه رند روكش ها زير ميكروسكوٍ تعيين دقيق نقاط اندازه

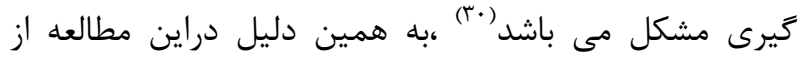
نيز جهت بررسى تطابق لبه اى استفاده شد.ميانخين AMO شولدر به ترتيب(Q مى باشد كه نسبت به كروه شولدر با ميانكين(•9/9 صورت معنى دارى كمتر مى باشد.بنا به اظهار شيلينبرى و (d: marginal opening; D: the ) d=D sinm فرمول distance by which a crown fails to seat and $\mathrm{m}$ : the acute angle of the margin) هر جه زاويه مارجين روكش با زاويه بيشترى ختم شود(شولدر) احتمال ايجاد مارجينال گٍَ بيشتر شده و اگر فاكتور سمان را هم در نظر بخيريم مقدار گَ بيشتر هم مى شود. (rV) در مطالعه

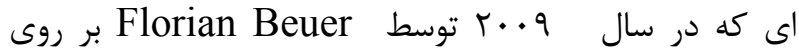
كويينگ هاى زيركونياى ساخته شده توسط Cercon انجام

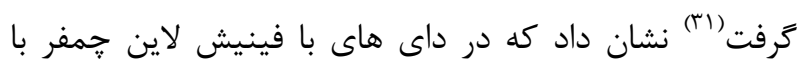

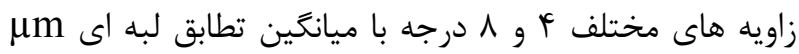

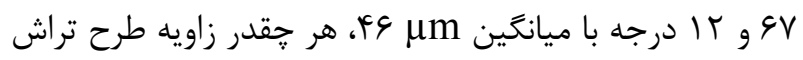
ما بيشتر باشد تطابق لبه اى روكش افزايش بيدا مى كند.

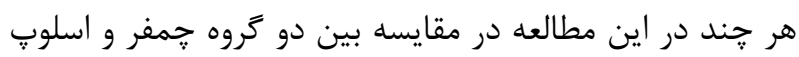
شولدر تفاوت معنى دارى ديده نشد،اما در كروه جمفر تطابق لبه اى بهترى ديده شد،دليل اين يديده مى تواند به محدوديت هاى دستگاه هاى فعلى در اسكن كردن زاويه هاى تيزاگزيوجينجيوال كه در حالت جمفر نسبت به اسلوبٍ شولدر

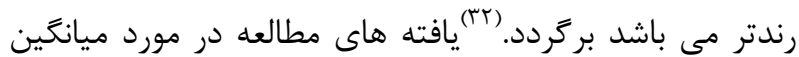

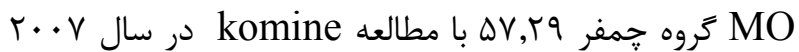

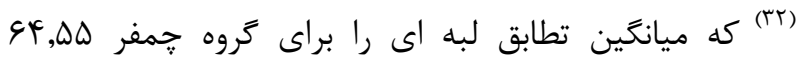

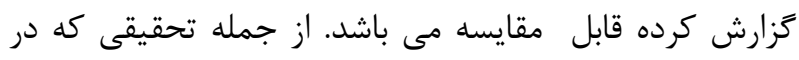

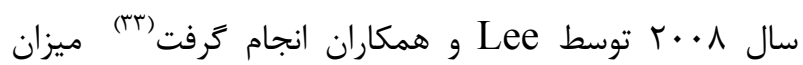
مارجينال گٍ در طرح تراش راديال شولدر به عمق الميلى

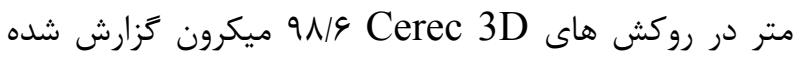

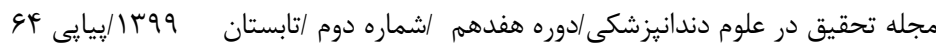


تراش chamfer به محدوده يذيرفته شده rD)ADA تا مه

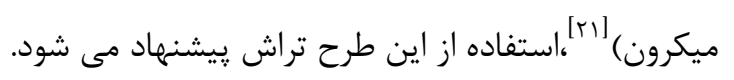

\section{References:}

1-Tsalouchou, E., et al. Fatigue and fracture properties of yttria partially stabilized zirconia crown systems. Dent Mater (2008): 24(3); 308-318.

2-Alshahrani, F.A., et al. A load-to-fracture and strain analysis of monolithic zirconia cantilevered frameworks. The Journal of prosthetic dentistry (2017): 118(6); 752-758.

3-Rieth PH, R.J., Naumann AW. Fabrication and flexural strength of ultrafine-grained yttria-stabilized zirconia. Am Ceram Soc Bull (US) 1976:55.

4-Subasi, G., et al. Evaluation of marginal fit of two all-ceramic copings with two finish lines. European journal of dentistry (2012): 6(2); 163.

5-Sulaiman, T.A., et al. Effect of different treatments on the flexural strength of fully versus partially stabilized monolithic zirconia. The Journal of prosthetic dentistry (2017): 118(2); 216-220.

6-Sailer, I., et al. Five-year clinical results of zirconia frameworks for posterior fixed partial dentures. International Journal of Prosthodontics (2007): 20(4); 383.

7-e Silva, J.S.A., et al. Marginal and internal fit of four-unit zirconia fixed dental prostheses based on digital and conventional impression techniques. Clinical oral investigations (2014): 18(2); 515-523.

8-Kokubo, Y., et al. Clinical marginal and internal gaps of zirconia all-ceramic crowns. Journal of prosthodontic research (2011): 55(1); 40-43.

9-Yeo, I.-S., J.-H. Yang, and J.-B. Lee. In vitro marginal fit of three all-ceramic crown systems. The Journal of prosthetic dentistry (2003): 90(5); 459-464.

10-Shillinburg, H., et al. Fundamentals of fixed prosthodontics. 3rd ed. Chicago: Quintessence (1997): 29--37.

11-Bugurman, B.B. and S.B. Turker. Clinical gap changes after porcelain firing cycles of zirconia fixed dentures. The journal of advanced prosthodontics (2014): 6(3); 177184.
تطابق داخلى روكش ها در اين مطالعه توسط دو شاخص گٍ Occlusal و Axial

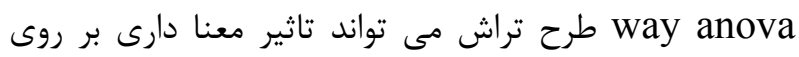

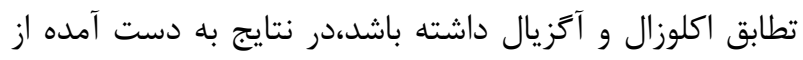
تست مقايسه اى Tukey HSD ميانگين گَ Occlusal و $\quad(r r, q) \pm \mid r \cdot r V / I \cdot r V \pm \uparrow q, q \cdot)$ مى باشد كه تفاوت معنى (I IF, دارى نداشته ولى هر دو گروه نسبت به گروه شولدر با ميانخين

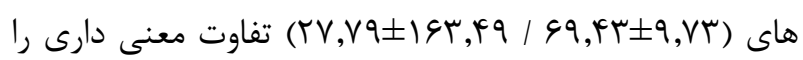
نشان دادند.با توجه به رابطه ى مستقيم اين شاخص ها با ميزان تطابق مارجينال اين نتايج قابل ييش بينى بود. اين تحقيق نشان داد كه ميزان AMO و MO طرح تراش زير 120 ميكرون بوده و از نظر كلينيكى مطلوب نهي

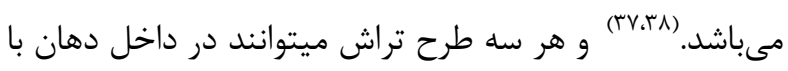

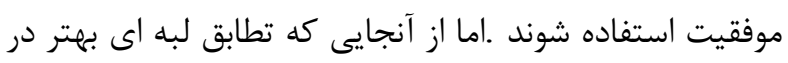

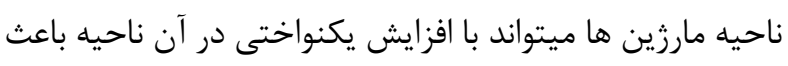

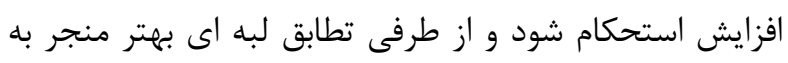

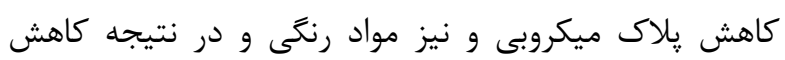

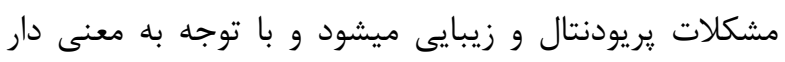

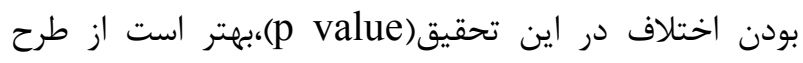

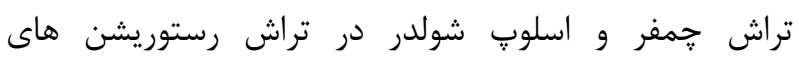
زير كونياى مونوليتيك استفاده شود.

$$
\begin{aligned}
& \text { نتيجه كيرى } \\
& \text { اين تحقيق نشان داد كه ميزان AMO و MO }
\end{aligned}
$$

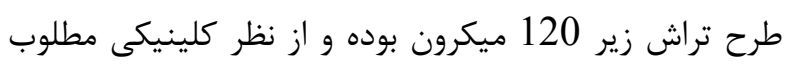

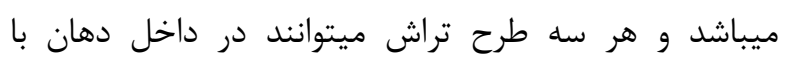

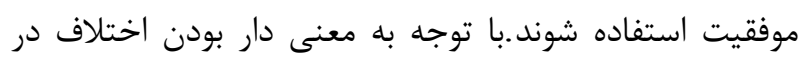

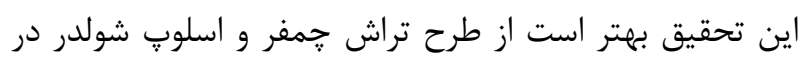

$$
\begin{aligned}
& \text { تراش رستوريشن هاى زيركونياى مونوليتيك استفاده شود،هر } \\
& \text { جند با توجه به نزديك بودن ميانگين تطابق لبه اى طرح }
\end{aligned}
$$


12-Quintas, A.F., F. Oliveira, and M.A. Bottino. Vertical marginal discrepancy of ceramic copings with different ceramic materials, finish lines, and luting agents: an in vitro evaluation. The Journal of prosthetic dentistry (2004): 92(3); 250-257.

13-Abduo ,J., K. Lyons, and M. Swain. Fit of zirconia fixed partial denture: a systematic review. Journal of Oral Rehabilitation (2010): 37(11); 866876.

14-Kale, E., et al. Effect of cement space on the marginal fit of CAD-CAM-fabricated monolithic zirconia crowns. The Journal of prosthetic dentistry (2016): 116(6); 890-895.

15-Akbar, J.H., et al. Marginal adaptation of Cerec 3 CAD/CAM composite crowns using two different finish line preparation designs. Journal of Prosthodontics (2006): 15(3); 155-163.

16-Comlekoglu, M., et al. Influence of cervical finish line type on the marginal adaptation of zirconia ceramic crowns. Oper Dent (2009): 34(5); 586-92.

17-Euan, R., et al. Comparison of the marginal adaptation of zirconium dioxide crowns in preparations with two different finish lines. J Prosthodont (2012): 21(4); 291-5.

18-Jalalian, E., R. Rostami, and B. Atashkar. Comparison of chamfer and deep chamfer preparation designs on the fracture resistance of zirconia core restorations. J Dent Res Dent Clin Dent Prospects (2011): 5(2); 41-5.

19-Gavelis, J., et al. The effect of various finish line preparations on the marginal seal and occlusal seat of full crown preparations. J Prosthet Dent (2004): 92(1); 1-7.

20-Di Iorio, D., et al. Effect of margin design on the fracture resistance of Procera all ceram cores: an in vitro study. J Contemp Dent Pract (2008): 9(2); 1-8.

21-Beuer, F., et al. Effect of preparation design on the fracture resistance of zirconia crown copings. Dent Mater J (2008): 27(3); 362-7.

22-Beuer, $\mathrm{F}$,.et al. Effect of preparation angles on the precision of zirconia crown copings fabricated by CAD/CAM system. Dental materials journal (2008): $27(6) ; 814-820$.

23-Tsalouchou, E., et al. Fatigue and fracture properties of yttria partially stabilized zirconia crown systems. Dent Mater (2008): 24(3); 308-318.

24-Alshahrani, F.A., et al. A load-to-fracture and strain analysis of monolithic zirconia cantilevered frameworks. The Journal of prosthetic dentistry (2017): 118(6); 752-758.
25-Rieth PH, R.J., Naumann AW. Fabrication and flexural strength of ultrafine-grained yttria-stabilized zirconia. Am Ceram Soc Bull (US) 1976:55.

26-Subasi, G., et al. Evaluation of marginal fit of two all-ceramic copings with two finish lines. European journal of dentistry (2012): 6(2); 163.

27-Sulaiman, T.A., et al. Effect of different treatments on the flexural strength of fully versus partially stabilized monolithic zirconia. The Journal of prosthetic dentistry (2017): 118(2); 216-220.

28-Sailer, I., et al. Five-year clinical results of zirconia frameworks for posterior fixed partial dentures. International Journal of Prosthodontics (2007): 20(4); 383.

29-e Silva, J.S.A., et al. Marginal and internal fit of four-unit zirconia fixed dental prostheses based on digital and conventional impression techniques. Clinical oral investigations (2014): 18(2); 515-523.

30-Kokubo, Y., et al. Clinical marginal and internal gaps of zirconia all-ceramic crowns. Journal of prosthodontic research (2011): 55(1); 40-43.

31-Yeo, I.-S., J.-H. Yang, and J.-B. Lee. In vitro marginal fit of three all-ceramic crown systems. The Journal of prosthetic dentistry (2003): 90(5); 459464.

32-Shillinburg, H., et al. Fundamentals of fixed prosthodontics. 3rd ed. Chicago: Quintessence (1997): 29--37.

33-Bugurman, B.B. and S.B. Turker. Clinical gap changes after porcelain firing cycles of zirconia fixed dentures. The journal of advanced prosthodontics (2014): 6(3); 177-184.

34-Quintas, A.F., F. Oliveira, and M.A. Bottino. Vertical marginal discrepancy of ceramic copings with different ceramic materials, finish lines, and luting agents: an in vitro evaluation. The Journal of prosthetic dentistry (2004): 92(3); 250-257.

35-Abduo ,J., K. Lyons, and M. Swain. Fit of zirconia fixed partial denture: a systematic review. Journal of Oral Rehabilitation (2010): 37(11); 866-876.

36-Ahmed, W.M., et al. Marginal discrepancies of monolithic zirconia crowns: the influence of preparation designs and sintering techniques. Journal of Prosthodontics (2019): 28(3); 288-298.

37-Goldin, E.B., et al. Marginal fit of leucite-glass pressable ceramic restorations and ceramic-pressedto-metal restorations. The Journal of prosthetic dentistry (2005): 93(2); 143-147.

38-McLean, J. The estimation of cement film thickness by an in vivo technique. Br dent j (1971): 131(107-111.

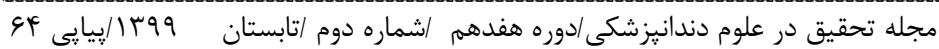

